\title{
Redefining the infrastructural response model: using an informational framework to proactively mitigate large-scale coastal events
}

\author{
D. G. King ${ }^{1} \&$ M. C. Lee $^{2}$ \\ ${ }^{1}$ 2kings Architecture, USA \\ ${ }^{2}$ School of Architecture, University of North Carolina at Charlotte, USA
}

\begin{abstract}
One of the major challenges facing coastal cities is how they prepare and respond to the devastating forces of natural events. Currently, after every major disaster, professionals step forward in an attempt to solve post-disaster problems. This "Response Model" proposes solutions that are aimed at addressing large-scale regional issues by crafting a comprehensive set of solutions that are designed for a small-scale implementable area. The problem with the Response Model is that comprehensive solutions are tailored to fit a specific set of site conditions that are unique to the place addressed. This means that application of the comprehensive solution is difficult at other locations and thus does not do enough to address the large-scale regional issue it initially sought to solve. In addition, there is not a systematic mechanism to enable the research and creative ideas generated to apply to future disasters. The authors believe that the solution is not a reinvention of the Response Model, but rather an augmentation of it. Merging the creation of ideas with an informational framework would enable the Response Model to be categorized and reapplied to different locations, thus becoming a more useful tool. The authors therefore propose a "Proactive Model" that could be implemented by collecting the comprehensive solutions after each disaster response, separating each into its smaller micro-sized solution parts, then qualifying those parts based on a set of criteria. The solution parts could then effectively be recombined to form multitudes of new unique comprehensive solutions. This system would both allow a reconfiguration of solution parts to fit other unique places but also create a suppository of creative infrastructural ideas that could be utilized as an idea library for designers as well as government entities.

Keywords: critical infrastructure, information system, disaster recovery, disaster mitigation, coastal planning.
\end{abstract}




\section{Introduction}

One of the major challenges facing the future will be how cities deal with large scale infrastructural issues such as drought, overcrowding, flooding, and sea level rise. These infrastructural challenges pose a significant risk to both human life and asset values throughout the world. The impact of doing nothing would be catastrophic. In 2005, according to a study conducted by the Organization for Economic Co-operation and Development ("OECD") on the effects of flooding and sea level rise, over 40 million people were exposed to a 1-in-100year

flooding event in major urban areas and this represented assets at risk of US \$3 trillion, or 5\% of global GDP. By 2070, the estimated population at risk would rise to 150 million and assets exposed to US \$35 trillion [9]. These infrastructural needs are too large to ignore.

Despite this fact, the scale of the problems faced prevents proposed solutions from becoming actionable. This is due to the realities of politics on a large regional and national scale, economics, and even cross-professional cooperation that often hinder the formation and acceptance of creative, feasible solutions. These realities have shaped a system that needs actionable solutions yet fails to implement a structure that would allow solutions to be actionable. Currently, this system is designed such that most comprehensive, inter-disciplinary solutions are reactionary, being prompted after a major destructive event occurs, instead of before. That is not to say that solutions are not proposed prior to events occurring, just that most of these proposals are conceived by single disciplines and often lack a truly comprehensive approach which ultimately hinders their implementation. For example, engineers address infrastructural challenges from a more technical approach, while architects deal with more societal and ecological issues that often force their solutions into a more utopian genre. While this generalizes the solutions of both those groups, it is safe to say that addressing something such as economic feasibility should be left to financially trained professionals, as should the political complexities be left to political scientists, as well as urban and regional planners. Therefore, a truly comprehensive approach involves all these disciplines producing a single solution. Currently, this type of approach is utilized only as a response to a major disaster.

This comprehensive methodology has been utilized in part directly after the disaster that Hurricane Sandy wrought on the northeast United States in fall of 2012. The devastation that was caused to the region in general, but specifically the metro-New York area, prompted a national call to action. Every governmental body, including the federal government, sought potential ideas to mitigate the effects of sea level rise, flooding, and surge. Several design competitions emerged, requesting proposals for solutions to the infrastructural dilemma. Competitions hosted by the Museum of Modern Art (MoMA) in New York, Rebuild by Design, and Far Rockaways were a few of the larger competitions that garnered international entries. These competitions narrowed the entries from the hundreds to a small handful of teams that comprised architects, engineers, planners, urban designers, landscape architects, and financial analysts. These multi-disciplinary 
teams did extensive research on the large-scale regional issues, and then proposed interventions on a more manageable, implementable, and fundable scale. This methodology is a good evolution over the past single-disciplinary responses, however this "Response Model" is still reactive in nature. Furthermore, given that each reaction addresses a specific, unique place makes application on a broader scale more difficult. The lack of a mechanism to enable the research and creative ideas generated through these comprehensive solutions to apply to future places and future disasters means that professionals reinvent the wheel every time. The Response Model does not learn from the previous Response Models and thus the applications are minimized to the small-scale, failing to address the largerscale issues they sought to solve initially.

The question then becomes, how do comprehensive solutions become more proactive, allowing professionals and decision entities to access, understand, and apply them to other unique places? The solution is not a reinvention of the Response Model, but rather an augmentation of it. The Response Model is a good system for producing high quality, creative, forward thinking, and comprehensive ideas. It harnesses public energy in the wake of a disaster and creates an organized system through which multi-disciplinary teams can group to develop solutions. Merging the creation of ideas with an informational framework such as GIS would enable the Response Model to be categorized and reapplied to different locations, thus becoming a more useful, "Proactive" tool.

\section{Evolution of response methodology}

Throughout history the model that professionals have followed when putting forth proposals for change could be referred to as a Silo-Solution, whereby their proposed solution to a problem is made using just their acquired or gathered knowledge. The problem with this Silo Model is that the problem solver attempts to solve the problem using only one lens or filter, their own. The solution is proposed without having had the feedback and input from those that are professionals in related fields. We can see this Silo Model used in responses to most major infrastructural challenges in the United States as well as abroad. Hurricane Katrina in 2005 is a perfect example of a situation where architects, engineers, and other professionals worked separately in crafting solutions to the problems left in hurricane Katrina's wake. The inevitable problem is that the solutions are not comprehensive enough to answer all the issues involved in truly solving the problem. This happened again in 2009 when the American Society of Civil Engineers ("ASCE") outlined the risk facing New York regarding surge and flooding from a major storm and the Museum of Modern Art ("MoMA") in New York joined with PS1 Contemporary Art Center ("PS1") to host several architects in residence to address the issue of sea level rise. While both of these produced compelling and innovative ideas, none were complete enough to fully answer financial and political feasibility and were ultimately ignored.

However, after Hurricane Sandy hit, and the northeast was shut down, including New York City, large amounts of attention were then placed on solving sea level rise and protecting against future storms that produce the types of surge 
and flooding that was experienced. Everyone, including the President of the United States made this a priority. At this point, a new model of disaster response was forged, what was referred to as the Response Model in my Introduction. This new approach may have been due to the failures of previous disaster responses, the multi-firm approaches that the ASCE and MoMA PS1 had used, the success of the Highline project model in New York of a multi-disciplined approach using everyone from the community to financial analysts to propose actionable solutions, or the combination of all three, but there was a fundamental shift that took place in how we addressed major infrastructural problems.

In December 2012, the Hurricane Rebuilding Task Force was created by presidential executive order to ensure that the Federal Government continues to provide resources to support regional resilience, health, and prosperity. As part of that executive order, the Hurricane Rebuilding Task Force launches the Rebuild by Design (RBD) competition in June 2013. The competition was designed to promote both innovations through "regionally scalable but locally contextual solutions" as well as implement those ideas through both public and private funding sources, including special disaster grants set aside by HUD [11]. The design competition encouraged the use of the following fields: community building, social science, economics, ecology, hydrology, water safety, transportation, resilience, sustainability, project management, finance, arts, and graphic design. Rebuild by Design realized that if ideas were to be both innovative and implementable that teams had to have diverse and complimentary backgrounds in order to succeed. This inter-disciplinary cooperation was a very different type of approach that had not been used extensively in design competitions.

What makes the Rebuild by Design competition so unique is its requirement for implementation. While all design competitions want realistic proposals, Rebuild by Design from the outset put in place a methodology that would ensure realism. Their requirement on team make-up, setting up checkpoints through stages that would guarantee engagement with the site, community, and government, and then finally requiring teams to back up their own proposals with an implementation strategy guided a process that allowed for innovation and creativity, but at the same time honed that creative thinking into a plan that could be actionable. This should be the goal of all infrastructural solutions, innovation and implementation.

\section{Scaling ideas by creating collective memory: the new methodology}

Rebuild by Design provides the framework for innovative, implementable, and comprehensive micro solutions to the macro problem of sea level rise. Rebuild by Design's mission to produce locally contextual solutions that could be regionally scalable was a perfect competition model. Its goal was not to dictate that every solution proposed had to be effective regionally or even to force teams involved to scale their solutions up to solve the regional problem. They knew that in order to produce both innovative and implementable solutions that the teams needed to 
focus on the geographic details and local community needs. By focusing on a small, micro level, the teams were able to reach a depth of understanding and level of design detail that made the proposals meaningful. This competition model is one that should continue to be emulated as it allows for the combination of visionary and actionable solutions. Yet, the question remains, how do you scale these ideas to solve the regional issue, and even beyond, how do you scale these ideas to solve the same issues outside the region on a national or international level?

We tend to reinvent the wheel every time we address the same set of issues. While there is a need for customized solutions, the overarching issues associated with a problem are all the same. For large scale infrastructural issues it is about creating a solution that solves the long-term problem but creates enough benefit, direct and perceived, in the short-term to be fundable. This holds true as well in the challenge of sea level rise dealt with by the ASCE and MoMa PS1 in 2010 and then by Rebuild by Design and others in 2013, the underlying problems are all the same. When looking over the proposals and the design boards produced, an observer can see the threads of similarity running through each proposal. Each team spent months of time analyzing the same regional information, trying to understand the particulars of governance, and understanding how disaster mitigation should work. As mentioned earlier, their ability to focus on a localized area enabled their solutions to be specific and detailed enough to be implementable, yet in their specifics the quandary of how to promulgate and apply their solutions to other regions exists. It is imperative that we understand how to utilize their innovative small-scale solutions to re-address the large-scale macro issue, given that their solutions and research represent a significant body of knowledge and understanding regarding responding and mitigating sea level rise. This body of knowledge created addresses a set of best practices that can be assessed and analyzed to avoid re-creating the wheel every time.

The concern is that when we produce innovative, actionable solutions, such as what has occurred with Rebuild by Design, that we are able to utilize those solutions to benefit the largest possible area (scaling the idea) and that we catalog those solutions so they may be easily accessed by others as needed. That is not to say that currently there is not a wealth of innovative and implementable material in existence to help address infrastructural problems, but rather there is not a simple method for finding relevant projects and dissecting those projects to utilize what is needed out of them. For example, if someone wants to apply the knowledge developed by one of the ten teams in Rebuild by Design, they must go to the RBD website and download the presentation boards that the team produced. These boards which were scaled for viewing on a wall for a live audience must now be read and assimilated electronically. To complicate this, each of these solutions was produced to address a set of unique conditions, so now the viewer must assess each of the ideas for applicability to the locality they are working in as well. Beyond that, what happens if they need this information five years from now and the competition is now not in the news, how do they access the projects? If the concerned is sea level rise in Virginia, they are researching its unique area, governance system, ecological makeup, and then searching for any precedent that 
could have anything to do with solving those particulars. We are right back to reinvention of the wheel.

This cycle can be broken by creating a system that can access this knowledge, thereby creating collective memory. Creating infrastructural collective memory is similar to the creation of the Dewey Decimal System that enabled the categorization and relative locations of large amounts of information to be organized in libraries. Cataloging and creating a system of micro-level infrastructural ideas that can be accessed based on specific sets of criteria would enable more efficient sharing of good practices. This system would break larger solutions into categorized parts that would allow problem solvers to quickly access relevant projects that could then be evaluated for usefulness. This would allow information, knowledge, and expertise to be effectively shared. It would also enable non multi-disciplinary teams to effectively access other professions expertise on relevant projects and provide a source of contact outside their field of study allowing new projects to become more comprehensive. When this collective memory is paired with modern technology, such as databases and the internet, it creates a system for long-term sharing of ideas.

In order to illustrate the effectiveness of a collective memory database the authors analyzed, dissected, and compiled the infrastructural solutions proposed by all ten teams in the Rebuild by Design competition. The database, named reCoMbination, is designed to allow a user to query it based on several specific fields of information. It initially takes the ten proposals and divides them into a set of micro-sized components that make up the whole. This process of divergence allows the customized solutions proposed for a specific locality to become a set of generic components. These generic micro solutions are given qualities based on the issue they address, the type of solution, the geographic typology addressed and waterfront condition. Based on these qualities, these quantifiable micro solutions can then be effectively recombined to create new customized solutions. This allows the user to have a menu of choices (micro solutions) and to sift through that menu based on specific needs.

The four main qualifiers that were applied to the micro-solutions are not a comprehensive set. They were chosen given the data that was produced by the Rebuild by Design competition. It is not meant to be an exhaustive list, therefore there actually may be more typologies or different waterfront conditions, however, the qualities chosen were meant to serve as a framework for disaggregating and filtering the data. These qualifiers, which represent only one possible framework, are listed below:

- Issue addressed: flooding/surge;

- Geographic typology: ocean/barrier island/headland/inland bay/watershed/ urban;

- Waterfront condition: urban front/natural/transportation;

- Type of intervention: technical/ecological/economic/public safety/regional planning. 


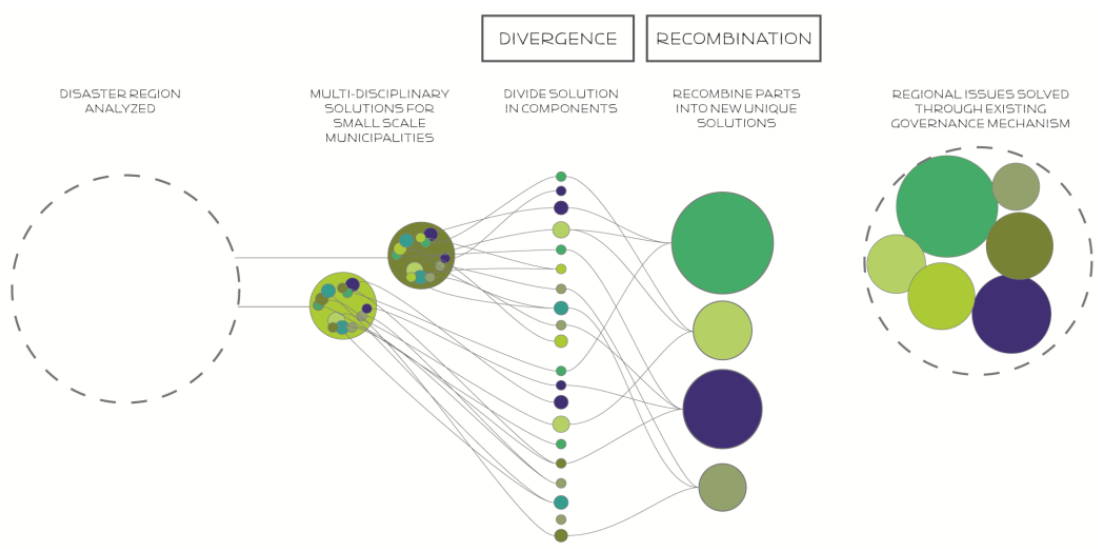

Figure 1: Disaggregation to reCoMbination.

The reCoMbination database is designed to effectively and efficiently disaggregate complex solutions and break them into more usable and less placespecific data. While it is conceptually not that different from a traditional precedent analysis, through its qualifiable methodology, it allows a designer to access a larger pool of precedents in a way that enables them to be filtered according to applicability. Its value is that it creates a method for the disaggregation of the place-specific, comprehensive solution into generic parts that can then be reassembled to form multiples of new comprehensive solutions, thereby ensuring a method to scale ideas to a broader area, as well as creating a long-term, proactive tool instead of the single-use, reactive one currently in place. The ability for well-designed solutions to be scaled and modified to benefit other unique places is paramount in solving the macro-level infrastructural issues. In addition, by gathering proposed and implemented solutions in a queryable database, ideas and knowledge of precedents and solutions may be freely shared, creating a powerful method of engaging any infrastructural issue and forming a valuable method for cataloguing collective memory. The ability to grow data organically utilizing technology could make this a powerful source of sharing information, thereby allowing future solutions with the benefit of historical knowledge and presumably allowing a more creative solution to be crafted.

\section{Case study: New Bern, North Carolina}

In order to illustrate the effectiveness of reCoMbination, the new "Proactive" methodological model will be utilized to extrapolate the post-hurricane comprehensive solutions for the greater New York region and reapply them to a new coastal location outside the Rebuild by Design area. This will allow for the separate components created by the Rebuild by Design competition to be reassembled to address a new unique place. New Bern, North Carolina has been selected as the case study location given its location and the authors' general familiarity with the location. 


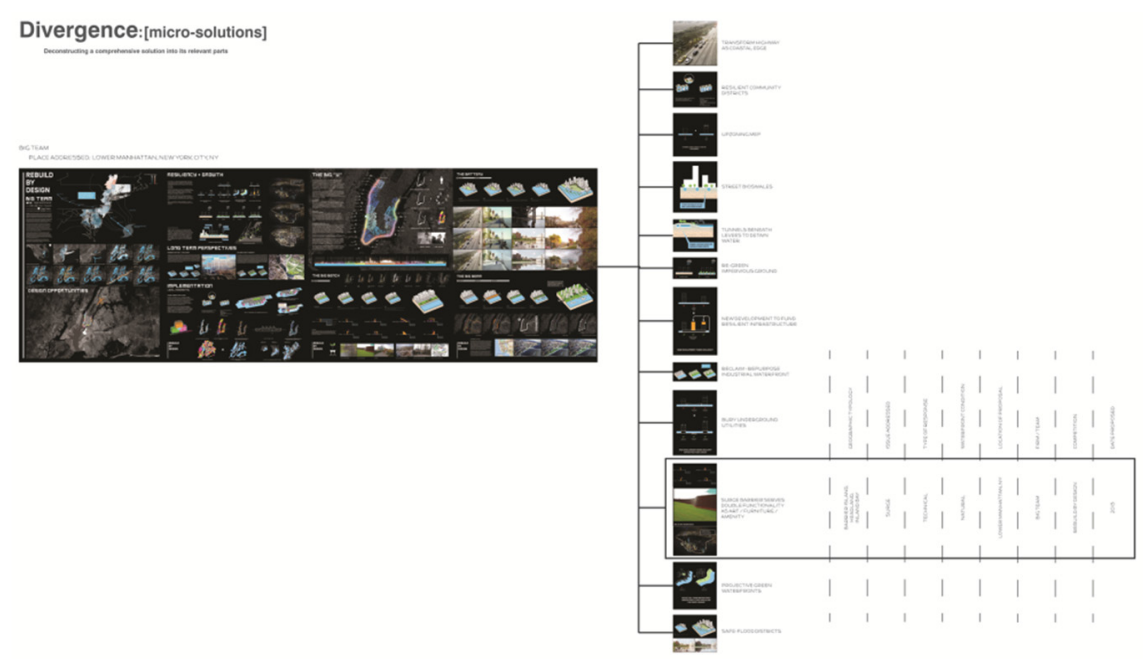

Figure 2: Disaggregation of BIG's rebuild by design proposal into micro solutions.

New Bern is a historical coastal town located in North Carolina's Inner Banks at the confluence of the Trent and the Neuse rivers. The second oldest town in North Carolina, it was settled in 1710 . The town comprises a 27 square mile area, of which four historical districts comprise approximately $2.5 \%$ but remain a vitally important draw for the city. New Bern served as North Carolina first continental capital and is famous for being the birthplace of Pepsi. Given its location on the Trent and Neuse rivers it is prone to heavy flooding due primarily to its low sea level elevation and the fact that it serves as the downriver coastal watershed for the surrounding geographic area. Urban development must be carefully regulated to conform and respect the existing historic character of the downtown. The waterfront conditions vary by location, but include single family residential, surface parking, park land, businesses, marinas, piers, and abandoned industrial.

The reCoMbination database contains 96 unique records after dividing the ten Rebuild by Design proposals into their more generic micro-level solutions, as can be seen from the images on the previous pages. This database has the ability to be filtered utilizing several qualitative characteristics that will narrow the potential relevance of the micro-level solutions. This can be seen on the left navigation pane of the web interface shown in figure 3.

In the case of New Bern, flooding is the main issue that must be addressed given its location further inland which protects it from frontline coastal surge. Geographic typology can be narrowed by selecting Inland Bay, Watershed, and Urban, all of which comprise the geographic conditions in the downtown and surrounding city. Finally, in order to separate the more traditional types of hard and soft interventions, the filters of ecological and technological will be selected. Given these filter selections, the number of potential micro-solutions is now twenty-seven. This list represents twenty-seven potential solutions for flooding in New Bern. These micro-solutions do not comprise a new solution in themselves, 


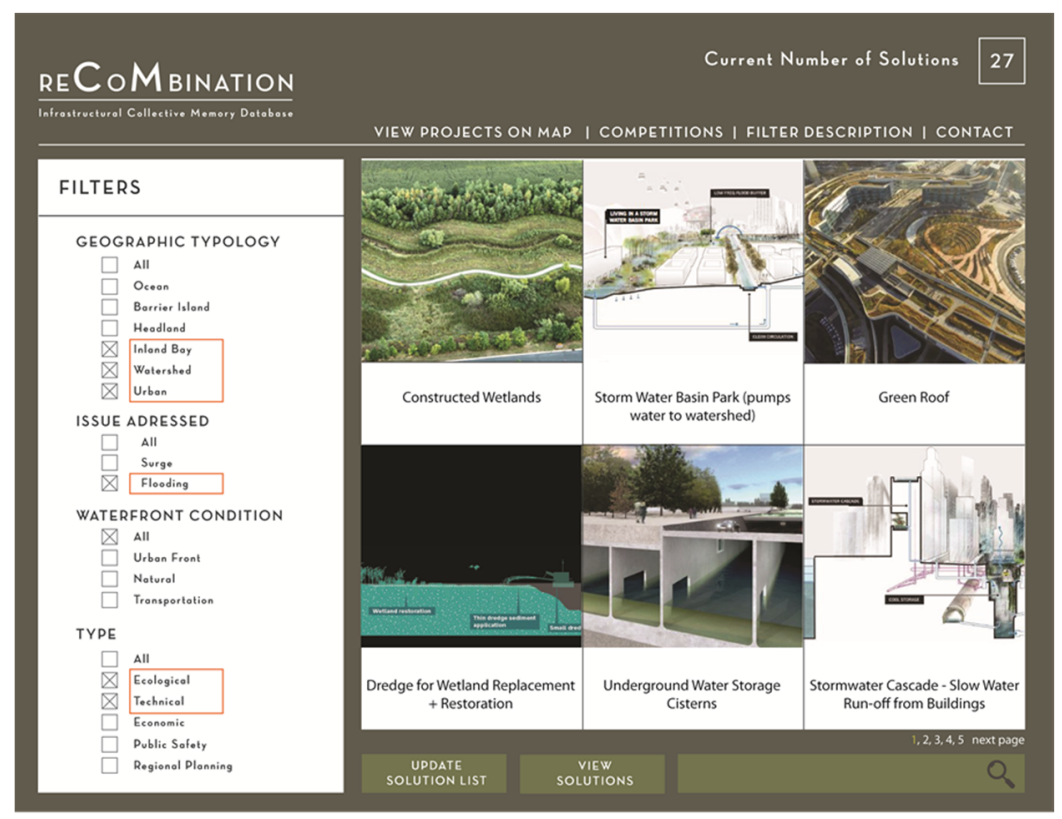

Figure 3: Filtering database of micro solutions using set criteria.

but must be further molded into a comprehensive solution that caters to the unique conditions within New Bern. What this list does do is allows the architect, engineer, or planner to quickly wade through all the ideas produced by ten multidisciplinary teams that each spent over a year studying the effects and responses possible to sea level rise and flooding, and filters those responses into ones that are most appropriate to the unique conditions in New Bern. This list of twentyseven represents a menu of choices that can further be selected and applied. It is a quick method of researching and finding applicable precedents to study, scale, and apply.

Of the filtered list of twenty-seven, eleven were found to be most applicable. The same filtering process applied to the hard and soft infrastructural flooding measures can also be done using the response types of Economic, Public Safety, and Regional Planning. After applying those filters the result is another seventeen potential solutions. Again, of the seventeen potential ideas, seven have the most direct applicability.

After the database has been filtered, these solutions may be selected one at a time, giving the user the ability to get greater level of details, including the website and contact information for the competitions, the team competitor make-up, as well firm websites and contact information. This enables the user to reach out directly to the relevant authors for more information and aids in the sharing of collective memory. This process of sifting through and narrowing the list and then applying the micro-solutions to create a new comprehensive solution took very 
little time leaving valuable time for other research methodology and community outreach. The comprehensive solution can graphically be viewed in figure 4 .

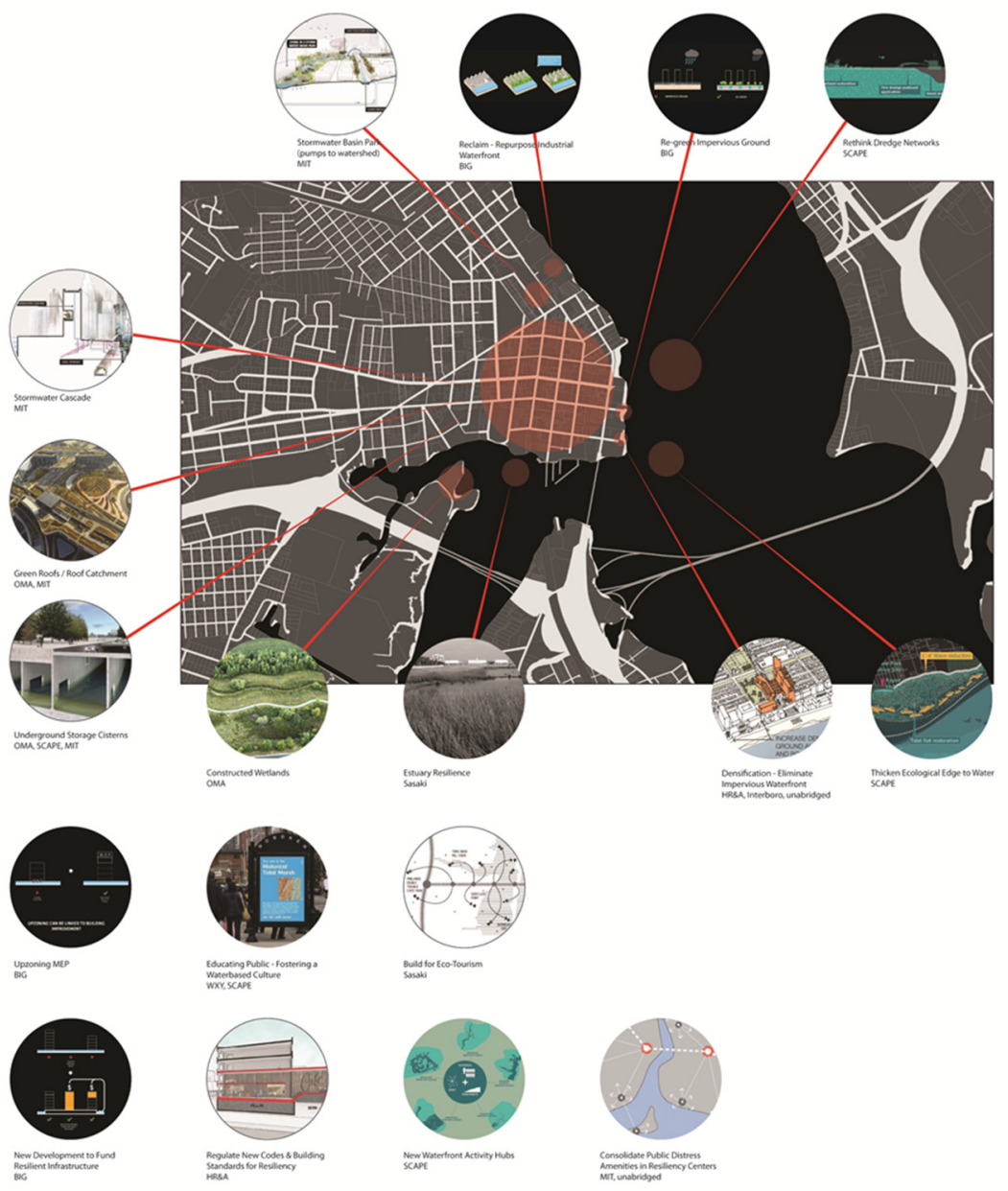

Figure 4: Filtered micro solutions recombine to form a new comprehensive solution.

\section{Conclusion}

The benefit of reCoMbination is the system it puts in place to proactively transform a single, one-off solution into multiples of new solutions that can be used to solve similar infrastructural issues in other unique places. It also serves as a clear and quick conveyance of collective information, thereby benefitting all infrastructural projects. One of the goals set at the start of the Rebuild by Design competition was that the solutions, although applied at the local level, could 
possibly be scaled to address regional issues. However, with no method in place for doing this, scaling a unique comprehensive design from one area to another area is very difficult. The reCoMbination database allows for the disaggregation of the comprehensive solution into generic parts that then can be reassembled to form a new comprehensive solution, thereby ensuring a method to scale the ideas to a broader area. It also allows ideas and knowledge generated reactively from a disaster to be shared and applied proactively in another region that faces the same threat but has not experienced the disaster first hand. While the duration and scope of this study prevented analysis beyond the Rebuild by Design competition, the ability to organically grow the information contained in the reCoMbination database to other competitions, design proposals, and built works would make it a powerful method of engaging any infrastructural issue and forming a valuable method for cataloging collective memory. The next step would be cataloging a greater number of proposed solutions and implemented projects to form a more comprehensive list of solution-parts that could have reapplied to solve major infrastructural issues. reCoMbination is the method that cities can use to begin addressing the larger infrastructural issues and with some cooperation, solve them proactively.

\section{References}

[1] Aquilino, Marie ed. Beyond Shelter: Architecture and Human Dignity. New York: Metropolis Books, 2011.

[2] Ballon, Hilliary, and Kenneth T. Jackson. Robert Moses and the Modern City. New York: W. W. Norton Company, 2007.

[3] FEMA. FEMA Mitigation and Insurance Strategic Plan 2011-2014. New York: FEMA, 2011.

[4] Foster, Kathryn A. “A Region of One's Own”. In Regional Planning in America: Practise and Prospect, by Ethan and Armondo Carbonell, eds. Seltzer, pp. 53-79. New York: New Lincoln Book Institute, 2011.

[5] Lin, Zhongjie. Kenzo Tange and the Metabolist Movement: Urban Utopias of Modern Japan. New York: Routledge, 2010.

[6] McCaffrey, Paul. US Infrastructure. Ipswich, Massachusetts: H.W. Wilson, 2011.

[7] Miles, Mike E., Gayle L. Berens, Mark J. Eppli, and Marc A. Weiss. Real Estate Development: Principles and Process. Washington, DC: Urban Land Institute, 2007.

[8] Mileti, Dennis S. Disasters By Design. Washington DC: Joseph Henry Press, 1999.

[9] OECD. Urban Infrastructure: Finance and Management. Paris: OECD, 1991.

[10] Padron, Dennis and Graeme Forsyth. NY-NJ Outer Harbor Gateway. Presentation, New York: Halcrow, 2009.

[11] Rebuild by Design. "Promoting Resilience Post-Sandy Through Innovative Planning and Design”. Rebuild By Design. 2013. 
320 Urban Water Systems and Floods

[12] Rohatyn, Felix G. and Everett Ehrlich. "A New Bank to Save Our Infrastructure". The New York Review of Books, October 8, 2008.

[13] Vanladingham, Kenneth E. "Municipal Home Rule in the United States". William and Mary Law Review, 1968: Volume 10, Number 2. 